\title{
8. Organoide: ein Fall für den Datenschutz? 1
}

\subsection{Umriss der aktuellen medizinischen Bedeutung}

Organoide sind dreidimensionale organähnliche Mikrostrukturen, welche aus verschiedenen Stammzellen in vitro hergestellt werden (Bartfeld/Clevers, 2018: 91; siehe auch Einleitung, Kap. 2.1). Die biologische Einordnung dieser künstlichen Zellgebilde ist an die körperliche Funktionalität des nachmodellierten Organs angelehnt (siehe auch Fagan, Kap. 4 für eine differenzierte Diskussion der wissenschaftlichen Modellfunktion von Organoiden). ${ }^{2}$ Sie eignen sich dazu, gewebespezifische Eigenschaften und zelluläre Kommunikationsprozesse nachzuvollziehen und ermöglichen ihr unmittelbares Studium. ${ }^{3}$ Des Weiteren können sie helfen, krankheitsspezifische Mechanismen oder Genmutationen, die Fehlbildungen oder Funktionsstörungen verursachen, nachzubilden sowie spezifische Behandlungen an ihnen zu testen. Organoide können auch mittels verschiedener Technologien wie der Genomeditierung (CRISPR/Cas) modifiziert werden, um den Effekt einer spezifischen Mutation zu untersuchen oder die $\mathrm{Mu}-$ tation eines bestimmten Patienten zu reparieren (siehe auch Teriyapirom/Batista-Rocha/Koo, Kap. 3.3). ${ }^{4}$ Bei der Erforschung von Infektionskrankheiten helfen sie, die menschliche Pathologie nachzuvollziehen. Auch für Arzneimitteltests sind sie vielver-

1 Die Autorin bedankt sich bei Frau Lisa Kaldowski, wissenschaftliche Mitarbeiterin an der Heidelberger Akademie der Wissenschaften, für die hilfreichen Diskussionen zum Beitrag sowie für die Bearbeitung der Fußnoten.

2 Neben neuronalen Organoiden werden $u$. a. auch Nieren, Leber oder Atemwegsorgane durch solche Gebilde dargestellt (Rowe/Daley, 2019: 380; Lancaster/Knoblich, 2014: 1247125).

3 Im Differenzierungsprozess ahmen sie die Zellgruppierung, räumliche Ausdifferenzierung und Funktion der Zelltypen in den Organen nach. Anschließend zeigen sie vergleichbare Reaktionen wie die Lebendorgane, sind aber an die Blutversorgung des (menschlichen) Körpers nicht angeschlossen und besitzen keine volle Funktionsfähigkeit (Deutsches Referenzzentrum für Ethik in den Biowissenschaften, 2020; Europäisches Patentamt, 2020; Rossen et al., 2020: 101052; Lancaster/Knoblich, 2014: 1247125, 283; Sachs et al., 2019: e100300).

$4 \mathrm{Zu}$ diesen Verwendungsmöglichkeiten vergleiche: Artegiani et al., 2020: $321 \mathrm{ff}$. 
sprechende Modelle und könnten sogar helfen, Tierversuche zu reduzieren (Mörl, 2019: 62 ff.; Europäisches Patentamt, 2020; Lancaster/Knoblich, 2014: 1247125-7; siehe auch Interview mit Clevers, Kap. 2.2, für eine optimistische Einschätzung dessen). Aktuell erweisen sich Organoide bei der Bekämpfung von SARS-COVID-19 als hilfreich, insofern sie bei der Erforschung von Medikamenten für die Modellierung bestimmter Krankheitsabläufe herangezogen werden können (Max-Planck-Gesellschaft, 2020). In diesem Zusammenhang können Biobanken menschlicher Organoide dazu beitragen, die angewandte Forschung auszubauen und zu beschleunigen (van de Wetering et al., 2015: 933 ff.; Boers et al., 2016: 940). In Form von Organoiden können die entsprechenden Zellen von Patienten zunächst vermehrt, danach eingefroren und wieder aufgetaut und damit langfristig im Labor gelagert werden, um beispielsweise Toxizitäts- und Medikamentenstudien patientengruppenspezifisch durchzuführen (Sachs et al., 2019: e100300). Darüber hinaus könnten Organoide zukünftig in der regenerativen Medizin die Basis für transplantierbares Gewebe bilden. ${ }^{5}$

Zurzeit werden Organoide in der Krebsforschung besonders intensiv verwendet, denn auch von Patienten stammende Gewebetumoren können als Modell für (Tumor-)Organoide etabliert werden (Ubink et al., 2019: 1410 ff.; siehe auch Kretzschmar, Kap. 3.4). Diese patientenspezifischen Organoide (,patient-derived organoid“, PDO) gleichen in ihren genetischen Merkmalen den Patienten(-tumoren), von denen ihr Ursprungsmaterial stammt (Ubink et al., 2019: $1410 \mathrm{ff}$.; Amato et al., 2020: 835; Boers, 2019: 133). Ihre Genauigkeit für die Vorhersage des Behandlungserfolgs und des Nichterfolgs ist sehr hoch (Wolf, 2020). Somit kann jedes PDO als eine Art kleiner „Patientenversuch“ angesehen werden (Bartfeld/Clevers, 2018: 91). Für die medizinische Forschung und die Entwicklung neuer Behandlungsmethoden - die Etablierung neuartiger Krebsmedikamente inbegriffen - ist es von größter Bedeutung, dass Organoide unter Berücksichtigung spezifischer genetischer und genomischer Merkmale generiert werden können und diese im Labor bei den Untersuchungen mitberücksichtigt werden können. ${ }^{6}$ Künftig könnten sie dazu beitragen, den Patienten individualisierte und stra-

5 Sie können aus kleinen Mengen von Spenderzellen gezüchtet, in vitro vermehrt und differenziert werden und autologe (vom Patienten selbst stammende) Zellen oder autologes Gewebe für Transplantationen liefern. In den autologen Organoidtransplantationen könnten krankheitsauslösende Mutationen - wie bereits oben geschildert - mithilfe verschiedener gentechnischer Methoden in vitro korrigiert werden, bevor die Organoide transplantiert werden (Lancaster/Knoblich, 2014: 1247125-7f.; Boers et al., 2019: 132).

6 Zudem gewährleistet ihr kurzer Produktionszyklus eine gute Versorgung während der Arzneimittelprüfung (Kim et al., 2019: 7). 
tifizierte, somit für sie am besten geeignete Behandlungen zu ermöglichen, und könnten damit zu einem wichtigen Baustein in der personalisierten Medizin werden. ${ }^{7}$

Diese medizinische Bedeutung von Organoiden als spezifischer Modellgegenstand deutet auf die Wichtigkeit ihrer Erfassung als besondere Daten- und Informationsquellen hin, die durch Überschneidungen vor allem in ihrer genetischen Ausstattung mit der des Patientenmaterials nahegelegt wird und die ihre Bedeutung für die translationale Medizin und ihrer Lagerung für längere Zeiträume in Biobanken untermauert (Maher et al., 2019: 242f.). Aus diesem Grund werden Organoide in dem vorliegenden Kapitel aus einer datenschutzrechtlichen Perspektive analysiert (für die Darstellung der deutschen Rechtslage im Allgemeinen siehe Taupitz, Kap. 7).

Die leitenden Fragestellungen in diesem Beitrag sind, ob und inwiefern datenschutzrechtliche Herausforderungen bezüglich eines Arbeitens mit Organoiden im Behandlungskontext sowie bezüglich einer (Weiter-)Verwendung für Forschungszwecke entstehen. An erster Stelle wird den Bedingungen für den Einschluss von Teilnehmern in Forschungskontexte und im Kontext klinischer Studien nachgegangen. Im Zusammenhang mit der Einbeziehung von Teilnehmern und der Entnahme ihrer Stammzellen aus Gewebeproben sowie mit ihrer Verarbeitung für datengeleitete Untersuchungen an Organoiden kommt der informierten Einwilligung eine zentrale Rolle zu. Nicht zuletzt erfordern Organoidbiobanken die Entwicklung spezifischer Verfahren zur Einwilligung, die dem breiten Spektrum von Weiterverwendungszwecken gerecht werden. Der Aufbau von Organoidbiobanken kann als ein Ansatz betrachtet werden, der sich von der mittlerweile herrschenden Herangehensweise in der digitalisierten Medizin unterscheidet: Der Patient wird nicht außerhalb der Klinik und in seinem Alltagskontext mithilfe verschiedener Technologien untersucht und beobachtet, wie es in der Telematik und bei selbstgesteuerten Gesundheits-Apps der Fall ist und das aus dem Patienten eine „wandernde“, sozusagen bewegliche, Datenbank macht. Stattdessen werden einzelne Organe oder deren Störungen im Labor modelliert und dort im notwendigen Umfang abgebildet und untersucht, um die Ergebnisse danach ggf. in die Therapie und weitere Forschung einzubinden.

Für die oben genannten Fragestellungen wird die noch vergleichsweise junge Anwendungspraxis einschlägiger, unmittelbar geltender unionsrechtlicher Bestimmungen der Datenschutz-Grundverordnung (DSGVO) und die Umsetzung der Verordnung

7 Zu den Einschränkungen, auch bzgl. Abweichungen vom Originaltumor siehe Pitzschke (2017). 
in Deutschland durch das Bundesdatenschutzgesetz (BDSG) herangezogen. ${ }^{8}$ Zum Schluss wird die grundlegende Herangehensweise an die Stellung des Patienten bei einer Verarbeitung von Organoiden in Biobanken skizziert.

\subsection{Drei Szenarien aus der Perspektive des Datenschutzrechts}

Es sind grundsätzlich drei datenschutzrechtlich bedeutende Szenarien vorstellbar, die bei einer Arbeit an Organoiden Bedeutung erlangen können:

Das erste Szenario deckt den Fall ab, bei dem es zur Forschung an Organoiden aufgrund eines Behandlungsverhältnisses kommt. Dabei wird das Organoid im Rahmen einer Diagnose und Behandlung entwickelt. Zur Behandlung kommt ein Forschungsprojekt hinzu, im Rahmen dessen Patientendaten und sensible Daten, die aus dem Organoid und während seiner Untersuchung gewonnenen wurden, über den Behandlungskontext hinaus weiterverwendet werden. Wie kann diese Sekundärnutzung legitimiert werden?

Das zweite Szenario stellt einen Sachverhalt dar, in dem es allein um die Forschung an Organoiden geht. Im Anschluss an das primäre Forschungsvorhaben soll ein weiteres, sekundäres Forschungsvorhaben mit den gewonnenen Daten realisiert werden, das sich in seinen Zwecken vom ursprünglichen Vorhaben unterscheidet. Zum Zeitpunkt der Datenerhebung für die primären Forschungszwecke war das zweite Vorhaben noch nicht (vollständig) absehbar. Kann die ursprüngliche Einwilligung das zweite Vorhaben legitimieren?

Das dritte Szenario betrifft Organoidbiobanken, aus denen Organoide bzw. aus ihnen gewonnenen Daten für verschiedene Forschungszwecke zur Verfügung gestellt werden. Was gilt es zu beachten bei den Einwilligungen in der Biobankenforschung?

8 In der medizinischen Forschung werden in der Regel länderübergreifende Vorhaben angestrebt. Um für eine einheitliche Regelung zu sorgen, wird seit Längerem eine Lösung auf Bundesebene, häufig ausgehend von dem Bundesdatenschutzgesetz (BDSG), angeregt (vgl. statt vieler Krawczak/Weichert, 2017). Hierdurch soll die Forschungsinteraktion innerhalb von Deutschland aber auch nach außen erleichtert werden. Im vorliegenden Beitrag liegt der Schwerpunkt der Darstellung daher auf den Vorgaben der DSGVO und des BDSG, Regelungsbeispiele aus wenigen Ländern werden nur exemplarisch erwähnt, um ihre Relevanz für die Forschung zu verdeutlichen. Für die unterschiedlichen Anwendungsbereiche der Landeskrankenhausgesetze, Landesdatenschutzgesetze und des Bundesdatenschutzgesetzes für Forschungsvorhaben siehe statt vieler: Kühling/Buchner/Weichert DS-GVO Art. 9 Rn. 168-180. 


\subsubsection{Organoide aus der Behandlung in die Forschung}

\section{a) Die Rechtsgrundlage der Datenverarbeitung im Behandlungskontext}

Wenn Organoide zunächst im Behandlungskontext im Rahmen einer Beziehung zwischen Patient und Arzt gebildet und verwendet werden, ist für die hierfür notwendige Verarbeitung personenbezogener Daten die Rechtsgrundlage gemäß Art. 6 Abs. 1 DSGVO, für die Verarbeitung sensibler Daten gemäß Art. 6 Abs. 1 i. V. m. Art. 9 Abs. 2 DSGVO einschlägig. ${ }^{9}$ In der Regel werden für die Erstellung von Organoiden personenbezogene Daten in Form von Patientendaten benötigt - das Organoid selbst ist aber auch Träger sensibler Daten wie z. B. genetischer Daten. Um die Ergebnisse aus der Untersuchung des jeweiligen Organoids ggf. in die Patientenbehandlung zurückzuführen, soll ihre Zuordnung zum betroffenen Patienten ermöglicht werden, $d$. h. für die (Re-)Identifizierbarkeit anhand der einschlägigen Daten gesorgt werden. In einem solchen Fall wird die Verarbeitung nach Art. 9 Abs. 2 lit. h) i. V. m. mit Art. 6 Abs. 1 lit. b) DSGVO möglich. In diesem Kontext stellt der Behandlungsvertrag die Rechtsgrundlage dar und es wird vom Verbot der Verarbeitung besonderer Kategorien personenbezogener Daten zugunsten der Ausnahme für die medizinische Diagnostik sowie die Versorgung und Behandlung im Gesundheitsbereich abgewichen. Diese Ausnahme wurde im nationalen Recht in § 22 Abs. 1 Nr. 1 lit.b), Abs. 2 BDSG verankert. ${ }^{10}$ Als Rechtsgrundlage für die Verarbeitung besonderer Kategorien von Datensätzen, die für die Bildung und das Arbeiten mit Organoiden notwendig sind und ggf. für die im engen Sinne verstandene Datenverarbeitung für den Behandlungskontext nicht erhoben wurden, kann auf die ausdrückliche Einwilligung des Betroffenen nach Art. 9 Abs. 2 lit. a) i. V. m. Art. 6 Abs. 1 lit. a) DSGVO abgestellt werden (Arning, 2018: Rn. 179 ff.).

Die Ergebnisse von Medikamententests, die auf körpereigenen Zellen der Patienten basieren und in deren Medikationsplan mitberücksichtigt werden sollten, könnten beispielsweise auch für die elektronische Patientenakte (ePA) Bedeutung erlangen. Das Patientendaten-Schutz-Gesetz, das sich im laufenden Gesetzgebungsverfahren befindet und mit dem die neue ePA eingeführt werden soll, sieht nach $\S 341$ Abs. 2 lit. b)

9 Es geht also um eine herkömmliche Behandlung und daher gerade nicht um eine Datenverarbeitung in einer Notfallsituation, bei der die Ausnahme vom Verbot der Verarbeitung sensibler Daten im Schutz lebenswichtiger Interessen der Betroffenen zu finden wäre, Art. 9 Abs. 2 lit. c) DSGVO.

10 Auf Landesebene sind die Krankenhausgesetze sowie Universitätsklinika-Gesetze zu beachten, beispielsweise § 45 Abs. 1 Nr. 1 LKHG (BW); § 27 Abs. 2 LKHG (Bayern); § 24 Abs. 4 Nr. 1 LKHG (Berlin); Art. 15 Abs. 2 BayUniKlinG verweist auf § 27 LKHG (Bayern). 
SGB V n. F. vor, dass zu den Daten, die auf der ePA gespeichert werden können, u. a. auch der elektronische Medikationsplan hinzukommt. ${ }^{11}$

\section{b) Aus dem Behandlungskontext in die wissenschaftliche Forschung}

Art. 5 Abs. 1 lit. b) DSGVO besagt, dass Daten im Allgemeinen nicht für Zwecke weiterverarbeitet werden dürfen, die nicht mit den Zwecken vereinbar sind, die zum Zeitpunkt der Datenerhebung vorgesehen und den Betroffenen mitgeteilt wurden. Es wird aber darauf hingewiesen, dass die Weiterverarbeitung $u$. a. für wissenschaftliche Forschungszwecke nicht als unvereinbar mit dem ursprünglichen Zweck zu betrachten ist, wenn geeignete Schutzvorkehrungen gemäß Art. 89 Abs. 1 DSGVO getroffen werden. Grundsätzlich könnten demnach die Daten, die für die Erstellung eines Organoids - bzw. daraus gewonnen wurden -, das während der Diagnose, Behandlung und Therapie erstellt wurde, ohne Hinzuziehung einer weiteren Rechtsgrundlage für Forschungszwecke verwendet werden. Allerdings ist die Auslegung dieser Regelung nach Art. 5 Abs. 1 lit. b) 2. HS DSGVO umstritten. Kernpunkt des Streits ist, dass bei der schematischen Betrachtung die Interessen und Vertraulichkeitserwartungen der Betroffenen außen vor bleiben, was insbesondere unter Beachtung von Art. 7, 8 der Charta der Grundrechte der Europäischen Union problematisch sei (Schanz/Wolff, 2017: Rn. 1352). Auf jeden Fall ist die Privilegierung eng auszulegen und gilt nicht für fingiert-kompatible Zwecke. ${ }^{12}$ Dies bedeutet, dass die Fiktion nur zu dem Zweck greift, dass eine Verarbeitung zu nicht-kompatiblen Zwecken verboten ist. Die im Erwägungsgrund 50 DSGVO formulierte Folgerung, dass für zweckverträgliche Zweckände-

11 Gemäß § 358 Abs. 6 Nr. 2 SGB V n. F. ist die Nutzung des elektronischen Medikationsplans ebenso wie die Nutzung der ePA freiwillig. Ob ein Medikationsplan erstellt wird, ist davon abhängig, ob der Patient von seinem Anspruch aus § 31a SGB V Gebrauch macht, inhaltlich fällt der Medikationsplan in den Verantwortungsbereich des Arztes. Zugriffsberechtigte Leistungserbringer und andere zugriffsberechtigte Personen dürfen grundsätzlich auf personenbezogene Daten, insbesondere Gesundheitsdaten der Versicherten auf der ePA nach § 339 Abs. 1 SGB V n. F. zugreifen, soweit die Versicherten hierzu ihre vorherige Einwilligung durch zuvor erfolgte technische Zugriffsfreigabe erteilt haben. Nach § 359 Abs. 2 SGB V n. F. ist der Zugriff auf den elektronischen Medikationsplan nach § 334 Abs. 1 Nr. 4 SGB V n. F. abweichend von § 339 Abs. 1 SGB V n. F. ohne eine technische Zugriffsfreigabe der Versicherten zulässig, wenn die Versicherten auf das Erfordernis einer technischen Zugriffsfreigabe verzichtet haben und der Zugriff mit Einwilligung der Versicherten erfolgt (Bundesgesundheitsministerium, 2020). Vgl. die Stellungnahme des Bundesbeauftragten für den Datenschutz und die Informaationsfreiheit (BfDI) zu Folgen der Gesetzgebung des PDSG (BfDI, 2020).

12 Id. Rn. 413. Vgl. auch Erwgr. 50 DSGVO. 
rungen keine neue Rechtfertigung erforderlich ist, gilt nur für tatsächlich kompatible Zwecke.

Für die fingierten kompatiblen Zwecke der Forschung bleibt es überwiegender Ansicht nach bei dem Grundsatz, dass jede Verarbeitung einer Rechtsgrundlage bedarf, Art. 6 Abs. 1 DSGVO. Auch die Kompatibilitätsprüfung des Art. 6 Abs. 4 DSGVO ersetzt nicht die notwendige Rechtsgrundlage für eine Datenverarbeitung mit einem geänderten Zweck (Kühling/Buchner/Buchner/Petri DS-GVO Art. 6 Rn. 181 ff.).

Zwar stellt Art. 9 Abs. 1 DSGVO fest, dass die Verarbeitung von Gesundheitsdaten oder genetischen Daten generell verboten ist, erlaubt jedoch Art. 9 Abs. 2 DSGVO hiervon in den genannten Fällen abzuweichen. Zu den Abweichungsmöglichkeiten zählt auch Art. 9 Abs. 2 lit. a) DSGVO, welcher eine Ausnahme vom Verarbeitungsverbot vorsieht, wenn die betroffene Person ausdrücklich eingewilligt hat. Gemäß dem Kernprinzip der informationellen Selbstbestimmung hat diese Legitimation der Forschung durch die Einwilligung Vorrang vor anderen Erlaubnistatbeständen (vgl. Art. 8 Abs. 2 GRCh, Art. 5 Abs. 1, Art. 6 Abs. 1, Art. 9 Abs. 2 DSGVO). Eine (Weiter-)Verarbeitung zu Forschungszwecken ist auf Basis einer ausdrücklichen Einwilligung nach Art. 9 Abs. 2 lit. a) DSGVO zulässig und bewirkt, dass weitere Erlaubnistatbestände aus dem Unionsrecht oder dem Recht der Mitgliedstaaten nicht eingreifen. ${ }^{13}$ Vorgaben für die Rechtmäßigkeit ergeben sich aus Art. 9 Abs. 2 lit. a) und Art. 6 Abs. 1 lit.a) i. V. m. Art. 7 DSGVO. Allerdings ist es zum Zeitpunkt der Information der Patientinnen und Patienten über das Vorhaben nicht immer einfach, die Forschungsziele so zu definieren, dass die Maßstäbe einer ausdrücklichen Einwilligung erfüllt werden können (s. dazu unten Abschnitt 8.2.2). Eine weitere Möglichkeit, vom Verbot abzuweichen, bietet die rechtliche Privilegierung der Forschung auf nationaler sowie auf unionaler Ebene durch Rechtsvorschriften, die die (Weiter-)Verarbeitung von diesen Daten zu Zwecken der Forschung erlauben (gemäß Art. 9 Abs. 2 lit.j) DSGVO). Zur Begleitung dieser Forschungsvorhaben müssen gemäß Art. 89 Abs. 1 DSGVO geeignete Garantien für die Rechte und Freiheiten der betroffenen Personen implementiert werden, die auch sicherstellen, dass technische und organisatorische Maßnahmen bestehen, mit denen die Achtung der Datenschutzprinzipien wie die der Datenminimierung gewährleistet wird. Demnach sollen nur die Daten verwendet werden, die für die Erreichung der For-

13 Deutschland hat die Möglichkeit, die Legitimation der Verarbeitung sensibler Daten aufgrund einer expliziten Einwilligung einzuschränken wie durch Art. 9 Abs. 2 lit. a) DSGVO eröffnet, nicht genutzt (vgl. Krawczak/Weichert, 2017). 
schungsziele notwendig sind. Dies kann beispielsweise durch datenschutzfreundliche Voreinstellungen gewährleistet werden. ${ }^{14}$

Bei der Verarbeitung medizinischer und gesundheitsbezogener Daten zu Forschungszwecken ist in Deutschland auf Bundesebene § 27 BDSG anzuwenden. § 27 BDSG setzt die Öffnungsklausel nach Art. 9 Abs. 2 lit. j) DSGVO um und sieht eine Privilegierung der Datenverarbeitung für die Zwecke der wissenschaftlichen Forschung vor. Durch diese Regelung wird die Möglichkeit auf Bundesebene geschaffen, die Datenkomplexe, die für eine Forschung mit Organoiden notwendig sind und ggf. bereits im Behandlungskontext gesammelt und erstellt bzw. abgeleitet wurden, auch ohne Einwilligung zu nutzen. Voraussetzung dafür ist, dass die Verarbeitung für die im öffentlichen Interesse liegenden wissenschaftlichen Zwecke erforderlich ist. Mithin müssen die zu verarbeitenden Daten objektiv dem Zweck angemessen und erheblich sowie auf das für die Zwecke der Verarbeitung notwendige Maß beschränkt sein. ${ }^{15}$ Bei der Heranziehung einer forschungspriviligierenden Rechtsgrundlage müssen die landesrechtlichen Regelungen soweit einschlägig beachtet werden. Neben anderen Vorgaben unterscheiden diese am häufigsten zwischen krankenhausinterner und -externer Forschung bei der Ausgestaltung der anzuwendenden Regelungen. ${ }^{16}$ Auch bei der Übermittlung der Daten für Forschungszwecke können Krankenhausinteressen eine Rolle spielen. Bereits im Rahmen der Abwägung in § 27 BDSG könnte jedoch berücksichtigt werden, wo die Verarbeitung erfolgt und welchen Nutzen der Patient selbst daraus - in Verbindung mit der externen oder internen Eigenschaft der Durchführung der Forschung - ziehen kann.

Um die Patientendaten für Forschungszwecke weiterverarbeiten zu können, müssen nach $\S 27$ Abs. 1, Abs. 3 BDSG i. V. m. Art. 89 Abs. 1 DSGVO gewisse Mindestanforde-

14 EDPB, Guidelines 4/2019 on Article 25 Data Protection by Design and by Default, January 2020.

15 Moos et al. (2018): Kap. 5, Rn. 107.

16 Die Landesgesetze tragen zur Klärung der Rechtslage bei: Beispielhaft kann § 46 Abs. 1 Nr. 2a Landeskrankenhausgesetz Baden-Württemberg - LKGH (BW) herangezogen werden, demnach krankenhausinterne Forschungsprojekte Gesundheitsdaten ohne gesonderte Einwilligung verarbeiten dürfen. § 13, 25 Abs. 1 Landesdatenschutzgesetz Baden-Württemberg LDSG (BW) enthält eine vergleichbare Privilegierung der Forschung wie in § 27 BDSG. Ebenso kann § 25 Abs. 1 Landeskrankenhausgesetz Berlin - LKHG (Berlin) als Beispiel herangezogen werden: Für die krankenhausinterne Forschung ist in vielen Fällen eine Einwilligung nicht erforderlich, doch die Daten müssen gemäß Abs. 2 mindestens pseudonymisiert werden. Auch § 17 Landeskrankenhausgesetz Berlin - LDSG (Berlin) enthält eine vergleichbare Privilegierung wie § 27 BDSG. Im Gegensatz zu der Regelung der Weiterverarbeitung von Patientendaten für Forschungszwecke beinhalten die meisten Landeskrankenhausgesetze keine Erlaubnis zur Erhebung von Patientendaten für Forschungszwecke, § 25 LKHG Berlin stellt beispielsweise eine Ausnahme dar. 
rungen an ihren Schutz erfüllt werden. Die gleichen Anforderungen gelten für Art. 9 Abs. 2 lit.j) DSGVO. Mit $\S 27$ Abs. 3 BDSG und den relevanten Regelungen im Landesrecht wurde in Deutschland ebenfalls eine an die DSGVO angelehnte Garantie eingeführt. Da die berechtigten Interessen der betroffenen Person regelmäßig - insbesondere in der translationalen Medizin - gegen eine anonymisierte Datenverarbeitung sprechen werden, wird es im Falle der Organoidforschung auf eine pseudonymisierte Verarbeitung hinauslaufen, die Auflösung des Pseudonyms wird in der Regel dann erlaubt, wenn die Forschungszwecke dies erforderlich machen.

Des Weiteren soll angemerkt werden, dass §§ 303d, e SGB V die Einrichtung eines Forschungsdatenzentrums vorsieht. Das Forschungsdatenzentrum fördert u. a. die Entwicklung und das Zusammenfügen bestimmter Daten nach § 303b Abs. 1 SGB V von Versicherten wie Alter und Geschlecht, aber auch Vitalstatus und Angaben zum Sterbedatum. Dies kann auch für Forschungsvorhaben mit Organoiden, insbesondere für Vorhaben mit stratifiziertem Ansatz oder für Kohortenstudien zur Medikamentenund Behandlungsverträglichkeit Bedeutung erlangen. Nach § 303e SGB V gewährt das Forschungsdatenzentrum den genannten Einrichtungen, darunter Krankenkassen und Hochschulen, auf Antrag Zugang zu den Daten. Zudem sollten Versicherte gemäß § 363 SGB V n. F. ab 2023 die Möglichkeit haben, die in der ePA abgelegten Daten freiwillig pseudonymisiert und verschlüsselt der medizinischen Forschung zur Verfügung zu stellen, wofür eine ausdrückliche Einwilligung gemäß Art. 6 Abs. 1 lit. a) i. V. m. Art. 9 Abs. 2 lit. a) DSGVO erforderlich sein wird. ${ }^{17}$

$17 \S 342$ Abs. 2 Nr. 4 SGB V n. F.: Patienten können zu Forschungszwecken Zugang zu ihren Daten gewähren, sie willigen ein, dass die Daten nach $\S 363$ SGB V n. F. verarbeitet werden. Für die Festlegungen diesbezüglich der Gesellschaft für Telematik vgl. § 354 Abs. 2 Nr. 5 SGB V n. F. § 363 SGB V n. F. enthält die ausführlichen Regelungen zur Freigabe der Daten zur Verarbeitung zu wissenschaftlichen Forschungszwecken. Der Gesetzesentwurf stellt hierzu fest: „Behandlungsdaten können nur dann in der elektronischen Patientenakte gespeichert werden, soweit andere Rechtsvorschriften nicht entgegenstehen. Dadurch wird sichergestellt, dass der Anwendungsbereich anderer Rechtsvorschriften gewahrt wird. Beispielsweise enthält § 11 des Gendiagnostikgesetzes - GenDG Regelungen über die Mitteilung der Ergebnisse genetischer Untersuchungen und Analysen, insbesondere damit die betroffene Person nur im Arzt-Patienten-Verhältnis mit den Ergebnissen genetischer Untersuchungen und Analysen konfrontiert wird." Zwar ist das GenDG für die Verarbeitung genetischer Daten zu Forschungszwecken nicht anwendbar, aber wenn die genetischen Daten aus einem Behandlungskontext stammen, können sie nur dann in die ePA eingespeist werden, wenn der Patient sein Recht auf Nichtwissen nicht geltend gemacht hat. 


\section{c) Klinische Studien und wissenschaftliche Forschung}

Die EU-Verordnung über klinische Prüfungen (Clinical Trials Regulation, CTR) ${ }^{18}$ und die DSGVO gelten sowohl für klinische Studien als auch für die Weiterverarbeitung der Daten für die wissenschaftliche Forschung; wobei die CTR erst Ende 2021 in Kraft treten soll. Das Zusammenspiel der beiden Verordnungen kann für den Fall relevant sein, dass Organoide im Rahmen klinischer Studien entwickelt und verwendet werden. ${ }^{19}$

Während des gesamten Verlaufs einer klinischen Studie können zwei Hauptarten der Datenverarbeitung unterschieden werden, die Verarbeitung im Rahmen von Forschungsaktivitäten sowie die Verarbeitung für Zuverlässigkeits- und Sicherheitszwecke; zu Letzteren gehören beispielsweise die Durchführung der Sicherheitsberichterstattung und die Archivierung der Dokumentation. In seiner Stellungnahme 3/2019 vertritt der Europäische Datenschutzausschuss (EDPB) die Auffassung, dass die Verarbeitung zu Zuverlässigkeits- und Sicherheitszwecken in den Anwendungsbereich von Art. 6 Abs. 1 lit.c) DSGVO fällt, d. h. auf einer rechtlichen Verpflichtung beruht, und die Daten gemäß der Ausnahme nach Art. 9 Abs. 2 lit. i) DSGVO verarbeitet werden können, ${ }^{20} \mathrm{~d}$. h. bei Vorliegen eines öffentlichen Interesses im Bereich der öffentlichen Gesundheit. Voraussetzung ist, dass die Pflicht der Datenverarbeitung für diese Zwecke per Gesetz vorgeschrieben wird, und das Gesetz muss bestimmte Voraussetzungen erfüllen. ${ }^{21}$ Für diese Tätigkeiten im Rahmen einer klinischen Studie mit Organoiden ist somit keine Einwilligung in die Datenverarbeitung erforderlich (Bischoff, 2019: $270 \mathrm{f}$.); die Tätigkeiten sind notwendig, um die rechtlichen Verpflichtungen einzuhalten, denen der Sponsor oder der klinische Prüfer unterliegen.

Hinsichtlich der Verarbeitung, die ausschließlich Forschungsaktivitäten dient, schlägt der EDPB drei alternative Rechtsgrundlagen vor:

1. Eine im öffentlichen Interesse durchgeführte Aufgabe gemäß Art. 6 Abs. 1 lit.e) i. V. m. Art. 9 Abs. 2 lit. i) DSGVO, d. h. im öffentlichen Interesse im Bereich der öf-

18 Verordnung (EU) Nr. 536/2014 des Europäischen Parlaments und des Rates vom 16.04.2014 über klinische Prüfungen mit Humanarzneimitteln und zur Aufhebung der Richtlinie 2001/20/EG, OJ L 158, 27.05.2014: 1-76. Zur Definition der klinischen Studie vgl. Art. 2 Nr. 1 CTR. Zur Definition der klinischen Prüfung nach Art. 40 AMG vgl. Listl-Nörr (2018): § 40 AMG, Rn. 20.

19 Listl-Nörr (2018): § 40 AMG, Rn. 20.

20 EDPB (2019): Artikel 70 Absatz 1 Buchstabe b), Rn. 10 ff. Beispiele für diese Art der Verarbeitung sind die Durchführung von Sicherheitsberichten beispielsweise nach $₫ 40$ Abs. 2a S. 2 Nr. 3 AMG sowie nach Art. 41 bis 43 der Verordnung über klinische Prüfungen und bei Verpflichtungen zur Archivierung der Dokumentation über die klinische Prüfung die Archivierung der Stammdaten klinischer Prüfungen und der medizinischen Aufzeichnungen von Versuchspersonen gemäß § 40 AMG.

$21 \operatorname{EDPB}(2019)$ : Artikel 70 Absatz 1 Buchstabe b), Rn. $11 \mathrm{ff}$. 
fentlichen Gesundheit, oder i. V. m. Art. 9 Abs. 2 lit.j) DSGVO, d. h. zu Archivierungszwecken im öffentlichen Interesse, zu wissenschaftlichen oder historischen Forschungszwecken.

2. Aufgrund der berechtigten Interessen des für die Verarbeitung Verantwortlichen gemäß Art. 6 Abs. 1 lit.f) i. V. m. Art. 9 Abs. 2 lit. j) DSGVO.

3. Unter besonderen Umständen auf Grundlage der ausdrücklichen Zustimmung der betroffenen Person. ${ }^{22}$

Für die Feststellung, welche Rechtsgrundlage heranzuziehen ist, lässt sich folgendes festhalten:

Die Einwilligung nach der DSGVO hat nämlich nicht dieselbe Bedeutung wie die informierte Einwilligung nach der CTR. Die informierte Einwilligung in eine klinische Studie drückt die Bereitschaft der betroffenen Person aus, an einer bestimmten klinischen Studie teilzunehmen, nachdem sie über alle Aspekte informiert wurde, die für ihre Entscheidung zur Teilnahme bedeutend sind gemäß Art. 2 Nr. 21 CTR. Die informierte Einwilligung nach der CTR stellt damit weniger eine rechtliche Grundlage für die Verarbeitung personenbezogener Daten, sondern vielmehr eine Schutzmaßnahme für den Patienten dar. Der Unterschied zeigt sich auch darin, dass bei klinischen Prüfungen die Maßstäbe für die Freiwilligkeit der Einwilligung i. S. d. DSGVO nicht zu jeder Zeit erfüllt werden können; wie der EDPB hervorhebt. Dies sei der Fall, wenn die Teilnehmer keinen guten Gesundheitszustand aufweisen, zu einer sozioökonomisch benachteiligten Gruppe gehören oder sich in einer „Situation institutioneller oder hierarchischer Abhängigkeit" befinden. In solchen Fällen sind die anderen Rechtsgrundlagen für die Datenverarbeitung möglicherweise geeigneter und können, wo immer möglich, als Standardoption verwendet werden. Wenn als rechtliche Grundlage die informierte Einwilligung gewählt wird, muss eine besondere Bewertung der Situation des Patienten vorgenommen werden. ${ }^{23}$

In Bezug auf die sekundäre Verwendung von personenbezogenen Daten aus klinischen Studien, d. h. die Verwendung von Daten, die über den Rahmen des klinischen Studien- oder Prüfplans hinausgehen, erfordert die CTR die informierte Einwilligung des Teilnehmers. Diese informierte Einwilligung sollte vom Teilnehmer zum Zeitpunkt der Einwilligung in die Teilnahme an der klinischen Studie eingeholt werden. ${ }^{24}$ Auch diese Einwilligung sollte nicht mit der Einwilligung in die Datenverarbeitung nach der DSGVO verwechselt werden. Der EDPB erkennt an, dass eine neue Rechtsgrundlage für

$22 \operatorname{EDPB}$ (2019): Artikel 70 Absatz 1 Buchstabe b), Rn. 17.

23 EDPB (2019): Artikel 70 Absatz 1 Buchstabe b), Rn. 19 ff.

$24 \operatorname{EDPB}$ (2019): Artikel 70 Absatz 1 Buchstabe b), Rn. 29. 
diese Datenverarbeitung nicht erforderlich ist, sofern die Bedingungen von Art. 5 Abs. 1 lit. b) 2. HS DSGVO und Art. 89 DSGVO erfüllt sind. Dennoch kann die Einwilligung als Umsetzung des (medizin-)ethischen Fairnessgrundsatzes, der u. a. durch Art. 28 CTR vermittelt wird, gesehen und eingeholt werden. In dieser Funktion ist sie nicht dafür da, die Erfüllung von Datenschutzvorschriften zu gewährleisten, sondern dafür, die (medizin-)ethischen Anforderungen an die Forschung am Menschen zu erfüllen, deren Ursprung im Berufsrecht nachzuspüren ist. ${ }^{25}$

Teilnehmer an einer klinischen Studie können ihre informierte Einwilligung nach der CTR jederzeit widerrufen, wodurch sie sich aus der Studie zurückziehen können. Der Widerruf der Einwilligung in die klinische Studie nach der CTR beendet auch die Forschungsdatenverarbeitung, doch die Verarbeitung im Rahmen der Sicherheits- und Archivierungspflichten wird fortgesetzt. ${ }^{26}$ Diese Informationen sollten in die Einwilligungserklärung aufgenommen werden.

\subsubsection{Organoide in der Forschung und der Broad Consent}

Die Einwilligung nach der DSGVO muss im Allgemeinen für einen bestimmten Zweck erteilt werden. Wie oben angemerkt, ist es in der Forschung häufig herausfordernd, diese Zwecke mit der gebotenen Bestimmtheit zu definieren, denn es ist oft nicht möglich, den Zweck der Verarbeitung zum Zeitpunkt der Datenerhebung vollständig vorherzusehen und zu benennen. Häufig sind insbesondere in den Lebenswissenschaften die Forschungssituationen dynamisch und es kann vorkommen, dass die vollständige Zweckbestimmung der Datenverarbeitung schlechthin erst später, sogar vor dem Hintergrund der Arbeit mit den Daten möglich wird. Erwägungsgrund Nr. 33 DSGVO erweitert die allgemeinen Anforderungen um die Einwilligung in bestimmte Bereiche der wissenschaftlichen Forschung, wenn diese mit anerkannten ethischen Standards im Einklang stehen. Auch die jüngsten Empfehlungen des Ministerkomitees des Europarates (eine Internationale Organisation und Völkerrechtssubjekt) zum Schutz gesundheitsbezogener Daten lassen ausdrücklich eine breite Einwilligung („broad consent") für wissenschaftliche Forschungszwecke zu (Committee of Ministers, 2019). Durch die vielfältige Anwendung von Organoiden und die Möglichkeit ihrer schnellen Erstellung - was der Forschung insbesondere im Rahmen der Medikamentenprüfung zugutekommt - ist es tatsächlich vorstellbar, dass die relevanten erhobenen Daten für

25 WMA, Deklaration von Helsinki - Ethische Grundsätze für die medizinische Forschung am Menschen, verabschiedet von der 18. WMA-Generalversammlung, Juni 1964 Helsinki (Finnland); zuletzt revidiert durch die 64. WMA-Generalversammlung im Oktober 2013, Fortaleza (Brasilien).

$26 \operatorname{EDPB}$ (2019): Artikel 70 Absatz 1 Buchstabe b), Rn. 24. 
unterschiedliche, organoidbasierte Forschungen verwendet werden. Eine breite Einwilligung in die Organoidforschung würde bedeuten, dass ein Forschungsteilnehmer einwilligt, dass seine Daten für ein bestimmtes Spektrum zukünftiger Forschungsprojekte, ggf. mit unterschiedlichen Zwecken, verwendet werden können. Mit dieser Annahme geht die Frage einher: Wie kann eine solche Einwilligung zulässig umgesetzt werden? Sie darf zu keinem „blanket consent“ mutiert werden, sondern soll weiterhin als Grundlage für die Erlaubnis zur Forschungsdatenverarbeitung dienen.

Die Konferenz der Datenschutzaufsichtsbehörden (DAK) geht in ihrer Stellungnahme vom 3. April 2019 davon aus, dass nur dann, wenn die konkrete Ausgestaltung des Forschungsvorhabens eine vollständige Zweckbestimmung absehbar bis zum Zeitpunkt der Datenerhebung nicht zulässt, eine breite Einwilligung eingesetzt werden kann. ${ }^{27}$ Der EDPB wiederum stellt klar, dass Erwägungsgrund Nr. 33 DSGVO zwar einen flexibleren Ansatz erlaube, verabschiedet sich aber bei der Interpretation nicht gänzlich von der Idee einer spezifischen Einwilligung. Ein gut beschriebener Zweck sei nach wie vor erforderlich, was bedeutet, dass eine pauschal oder vage formulierte Einwilligung unwirksam bleibe, ${ }^{28}$ obwohl der Zweck der Forschung in der Einwilligung allgemeiner beschrieben werden könne (Schaar, 2017: 215). Auch die DAK stellt fest, dass es nicht vereinbar mit Erwägungsgrund Nr. 33 DSGVO wäre, wenn die Verwendung der erhobenen Daten in allgemeiner Form auf bestimmte Forschungsbereiche ausgedehnt würde. Das Erfordernis der Einwilligung nach Aufklärung setze zumindest voraus, dass das betroffene Forschungsprojekt durch die Einwilligungserklärung so genau wie möglich erfasst würde. Auf jeden Fall müssen, so der EDPB, Maßnahmen getroffen werden, um das Prinzip der spezifischen Einwilligung weiterhin zu respektieren, beispielsweise müssen größere Transparenz während der Datenverarbeitung gewährleistet und weitere Schutzvorkehrungen getroffen werden.

Eine Möglichkeit, die Forderung von Erwägungsgrund Nr. 33 DSGVO nach Einhaltung anerkannter ethischer Standards zu erfüllen, ist die Einholung der Genehmigung durch eine zuständige Ethikkommission für die Forschung. Ist dies vorhanden, würde das bedeuten, dass eine Person einwilligen könnte, an einem Forschungsprojekt teilzunehmen, das eine breit angelegte Datenverarbeitung im Rahmen von Untersuchungen an Organoiden gut beschreibt und Schutzvorkehrungen zur Minderung potenzieller Risiken und Schäden bei der Datenverarbeitung definiert, die durch die gut umschrie-

27 Datenschutzkonferenz (2019): Beschluss der 97. Konferenz der unabhängigen Datenschutzaufsichtsbehörden des Bundes und der Länder zu Auslegung des Begriffs „bestimmte Bereiche wissenschaftlicher Forschung" im Erwägungsgrund 33 der DS-GVO, 03.04.2019.

28 Article 29 Working Party, Guidelines on consent under Regulation 2016/679: $11 \mathrm{ff}$. 
benen Verarbeitungsprozesse bei noch unbekannten, zukünftigen Forschungsprojekten verursacht werden. Dem Erwägungsgrund Nr. 42 DSGVO kommt hierbei eine besondere Bedeutung zu: Damit die Einwilligung nachweisbar ist, sollte der betroffenen Person zumindest die Identität des für die Verarbeitung Verantwortlichen bekannt sein. Für die Nutzung der Daten für Sekundärforschung durch einen anderen Akteur verlangt Erwägungsgrund Nr. 33 DSGVO, dass der andere Akteur in der Lage sein muss, die Einwilligung nachzuweisen. Der andere Akteur muss zusätzlich dem Teilnehmer gegenüber identifiziert worden sein. Es bleibt allerdings dabei, dass während des Einwilligungsverfahrens nur der ursprüngliche, für die Verarbeitung Verantwortliche identifiziert werden muss. Bei späteren, noch unbekannten Datenempfängern (z. B. Forschern) kann es ausreichend sein, die Kategorien von Empfängern und damit die für die Verarbeitung Verantwortlichen und Auftragsverarbeiter zu nennen.

Eine zentrale Frage ist daher, wie spezifisch ein Broad Consent sein muss, damit eine Datennutzung im Rahmen verschiedener Forschungszwecke mit Organoiden abgedeckt wird. Dies wird sich regelmäßig gemäß den Zwecken der wissenschaftlichen Forschung entscheiden. Die Gestaltung des Rahmens für die sekundäre Nutzung aufgrund einer breiten Einwilligung sollte zudem durch zusätzliche Bemühungen der Verantwortlichen begleitet werden, wie z. B. durch die Festlegung von Kategorien der Datenverarbeitungsmethoden, Schutzmaßnahmen zur Minderung der Risiken dieser Methoden und, wie oben angemerkt, die Benennung der Kategorien potenzieller Datenempfänger, wenn diese noch nicht individualisierbar feststehen. Die DAK unterstreicht diese Korrekturmaßnahmen, die der Transparenz, der Datensicherheit und der Vertrauensbildung dienen, um die abstrakte Fassung des Forschungszwecks zu kompensieren sowie zusätzliche Garantiemaßnahmen zur Datensicherheit vorsehen sollten, um auch im Falle vergleichsweise unspezifisch bestimmter Forschungszwecke zum Zeitpunkt der Einwilligung etwaigen Verletzungen der Patientenrechte und Risiken für die Datensicherheit vorzubeugen. Als Maßnahme zur Förderung der Transparenz wird beispielsweise die Anwendung einer Nutzungsregelung oder eines Forschungsplans angesehen, der den Betroffenen zugänglich gemacht wird und der die geplanten Arbeitsmethoden und die Fragen, die Gegenstand der Forschung sein sollen, beleuchtet. Auch kann die Frage, weshalb eine detailliertere Konkretisierung der Forschungszwecke nicht möglich ist, auf das konkrete Forschungsprojekt bezogen ausführlich dokumentiert werden. Die Einrichtung einer Webseite zur Information der Studienteilnehmer über laufende und zukünftige Studien ist eine weitere, gängige Maßnahme. Zusätzliche Garantiemaßnahmen für die Datensicherheit beinhalten technisch-organisatorische Maßnahmen wie besondere Bestimmungen zur Beschränkung des Zugangs zu den gesammelten Daten. Zur Vertrauensbildung zählen beispielsweise 
die Einräumung einer Widerspruchsmöglichkeit vor der Verwendung der Daten für neue Forschungsfragen und Maßnahmen der dynamischen Einwilligung (,dynamic consent"), welche insbesondere bei Biobanken relevant wird. ${ }^{29}$ An dieser Stelle soll festgehalten werden, dass eine dynamische Einwilligung von der Funktionsweise her eine unterschiedliche Wirkung entfaltet: Parallel zur dynamischen Gestaltung des Forschungsvorhabens wird ihre Legitimationsgrundlage in Form der Einwilligung ganz nach Fortschreiten des Forschungsvorhabens zeitlich und inhaltlich aufgedröselt eine Prozeduralisierung in der Gestaltung der Rechtsgrundlage tritt dem Forschungsvorhaben bei. Die DAK hat ihr Einverständnis zu den Mustereinwilligungen der Medizininformatikinitiative gegeben, womit ein wichtiger Schritt zur Umsetzung des Broad Consent in Deutschland erreicht wurde (Medizininformatik-Initiative, 2019: 3 ff.; Medizininformatik-Initiative, 2020: 4 ff.). Bei der Arbeit der Aufsichtsbehörden ist eine hohe Akzeptanz zu erwarten.

\subsubsection{Biobanken für Organoide}

Um Organoide für die Forschung nutzbar zu machen, stellen sie hohe Anforderungen an Biobanken in Bezug auf Qualitätsstandards, personelles Know-how und die Datendokumentation (Amato et al., 2020: 839; Rowe/Daley, 2019: 378 f.). Bei der Bewältigung dieser Herausforderungen muss das herkömmliche Wissen über eingelagerte Biomaterialien und Proben mit Wissen zu den spezifischen biologischen Eigenschaften der Organoide erweitert werden, um die Erstellung und Haltung dieser Entitäten und die Forschung an ihnen zu ermöglichen.

Neue Untersuchungstechnologien und -methoden in der Biomedizin können die Organoidforschung bereichern, indem sehr gezielten biologisch-medizinischen Fragestellungen nachgegangen werden kann (z. B. mithilfe der Sequenziertechnologie). Hierfür wurden in den letzten Jahren durch neue Technologien und Methoden beachtenswerte Möglichkeiten eröffnet. Zudem sind die Möglichkeiten der Verarbeitung und Verknüpfung von Daten für die Forschung aus verschiedenen Quellen in den letzten Jahren flexibler geworden. In der Konsequenz können Untersuchungsgruppen von Studien und Forschungsteilnehmern zunehmend variabel bestimmt werden. Des Weiteren beruht die Nutzung, Speicherung und gemeinsame Verarbeitung von Daten häufig auf Clouds, die es ermöglichen, Daten zusammenzuführen und ihre gemeinsame Verarbeitung kostengünstig voranzutreiben, statt lediglich auf innerinstitutionelle, gar ortsgebundene Arbeit zu bauen. Die zunehmende Einbindung datenbasierter Tech- 
nologien in die Forschung und Versorgung erweitert die Datenerfassung (z. B. um die Erhebung von Lebensführungswissen mithilfe von Gesundheits-Apps oder Wearables). Zugleich erhöht die Verfügbarkeit und Verknüpfung von Daten die Beweglichkeit des Wissens und die Zugänglichkeit zu den Forschungsmitteln und -instrumenten und verstärkt insgesamt die flexiblere Definition von Verarbeitungs- und Forschungszielen.

In diesem Kontext könnten Organoidbiobanken dazu beitragen, Erkenntnisse aus Biomaterialproben und Forschungsdaten zu verknüpfen. Die Datenverwendung und das Einflechten verschiedener Studienergebnisse kann bei Organoiden dynamisch umgesetzt werden. Die Studienergebnisse können patientenspezifisch an den Organoiden überprüft werden und so zu individuellen oder stratifizierten klinisch verwendbaren Erkenntnissen führen und in beiden Bereichen die Begründung und Nachweisbarkeit der Forschungsschritte stärken.

Neben den neuen Möglichkeiten und Chancen entstehen jedoch auch neue Herausforderungen auf rechtlicher Seite der biobankbasierten Forschung, insbesondere hinsichtlich der Einwilligung der Patienten in die Datenverarbeitung. Für die Gestaltung der Einwilligungsprozesse sind die Hinweise der Datenschutzkonferenz bzgl. der Rolle der dynamischen Einwilligung zu beachten, die als Korrektivmaßnahme im Falle einer breiten Einwilligung herangezogen werden kann (vgl. Abschnitt 8.2.2). Als zusätzliche Sicherungsmaßnahme zur Gewährleistung von Transparenz empfiehlt die DAK zu prüfen, ob das Arbeiten mit einem Dynamic Consent möglich ist (Datenschutzkonferenz, 2019). Ein Dynamic Consent könne eine verstärkte individuelle Kontrolle erleichtern, sodass die Teilnahme an Forschungsvorhaben mit den Proben und Daten mehr nach den Präferenzen und Interessen des Einzelnen erfolgen könne. Die Gewährleistung einer aktiven Rolle für den Patienten während der gesamten Arbeitskette der Probenund Datenanalyse entspräche den nationalen (Medizininformatik-Initiative, 2019: 3 ff.; Medizininformatik-Initiative, 2020: 4 ff.) und internationalen Empfehlungen. ${ }^{30}$

Künftig können die besonderen Forschungsmöglichkeiten an Organoiden zu weiteren Überlegungen bzgl. der Governance-Struktur von Biobanken führen. In der Folge könnten herkömmliche Governance-Modelle mit Modellen kombiniert werden, die

\footnotetext{
30 WMA: Erklärung von Taipeh (DoT) 2016: Die Erklärung von Taipeh will das Vertrauen in die Daten und Biobanken stärken und dadurch zugleich ihre Arbeitsbereiche und Umfang ausdehnen. Hierfür soll eine klassische informierte Einwilligung so weit wie möglich verwendet werden (Nr. $11 \mathrm{ff}$.). Diese wird ergänzt durch kontinuierliche ethische Überprüfung der Nutzung und Entscheidung, ob eine weitere Einwilligung erforderlich ist (Nr.19). Hinzu kommen weitere Vorschriften zu Transparenz und Verarbeitung (Nr. 20, 21) um einen einheitlichen Standard zu gewährleisten. Die Empfehlung erkennt große Datenszenarien, indem von „mehrfacher und unbestimmter Nutzung“ gesprochen wird (WMA, 2016; vgl. auch OECD, 2017).
} 
Teilnehmer in den gesamten Forschungsprozess aktiv einbeziehen - auf Grundlage einer stärkeren Prozeduralisierung des Einwilligungsvorgangs und auf Basis der individuell-subjektiven Datenschutzrechte der Betroffenen - und die Modalitäten der Teilnehmerzentriertheit klar ausdifferenzieren. In diesem Prozess können die verschiedenen digitalen Technologien sowie die Forschungsmethoden, die an translationalen Möglichkeiten ausgerichtet durchgeführt werden, als „Bindeglied“ bei der Zusammenführung der Modelle dienen.

\subsection{Schlussfolgerung}

Die technisch-biologischen Möglichkeiten der Datenverarbeitung und des Erkenntnisgewinns werden durch die normative öffnung zur Flexibilität der Datenverarbeitung in der Forschung unterstützt. Diese Flexibilität spiegelt sich auch in der Privilegierung durch die Rechtsgrundlagen der Datenverarbeitung sowie in den unterschiedlichen Ausgestaltungsmöglichkeiten der Einwilligung (Stichwort: Broad Consent, Dynamic Consent) wider. Gleichwohl werden in den Datenschutzregelungen Kontrollmöglichkeiten für die Betroffenen, Verpflichtungen der Datenverarbeiter sowie technische und organisatorische Sicherheitsmaßnahmen bestimmt und die ethische Begleitung der Forschung gestärkt.

Vor dem Hintergrund der legislativen Abwägung zugunsten der Forschung in den unionalen und nationalen Datenschutzregelungen sollen diese Kontrollmöglichkeiten und Maßnahmen die einzelfallorientierte Abwägung im Zuge der Rechtsanwendung steuern. Durch die weitere Etablierung der translationalen Medizin auf Basis der Forschung mit Organoiden werden Forschungsziele und Patientenwohl noch stärker miteinander verbunden, da die ethisch-juristisch gewährleistete Flexibilität in der Forschung zugunsten des Patienten geschieht. Diese Vorteile zeigen sich bei der Arbeit mit Organoiden vor allem an den translationalen Perspektiven, so beispielsweise bei den tumorspezifischen Medikamenten- und Behandlungsreaktionen, sodass die betroffenen Patienten große, reale Chancen haben, von der Forschung selbst zu profitieren, weil die Ergebnisse nicht nur Patientengenerationen nach ihnen begünstigen. In der Abwägung Forschungsfreiheit einerseits und entgegenstehende Patientenrechte andererseits, rechtfertigt sich dadurch auch die weitreichende Privilegierung ersterer. Bei der Verwendung von Proben und Daten müssen die Patientenrechte allerdings weiterhin geschützt werden, da es kein objektivierbares Patientenwohl gibt, das über die Persönlichkeitsrechte gestellt werden könnte, und den Patienten nicht die Möglichkeit genommen werden darf, selbst zwischen Gesundheitsbelangen und dem kontextab- 
hängigen Schutz eigener Daten dynamisch oder auch kleinteilig zu entscheiden - die dem subjektiven Wohl entsprechende Balance sollten sie selbst austarieren dürfen.

Durch diese Entscheidungsfreiheit werden die Patienten zu aktiven und frei handelnden Partnern der Forschung. Ethikkommissionen können in diesem Gefüge als prüfende Instanz eingebunden werden, um ein Ungleichgewicht in der nach den Kräfteverhältnissen doch asymmetrischen Beziehung zu verhindern, das zur Abgabe unfreiwilliger, aber auch uninformierter Einwilligungen führen könnte. Die Ausdehnung des Einwilligungsprozesses soll in Verbindung mit den Informationspflichten des Verantwortlichen, in Art. 13 und 14 DSGVO festgeschrieben, gesehen werden - schließlich dienen die individuell-subjektiven Rechte einer Operationalisierung des Grundrechts auf Schutz personenbezogener Daten und des Selbstbestimmungsrechts des Patienten. Letztendlich wird durch die beiden Mechanismen auch die Akzeptanz und Legitimation der Forschung gestärkt, in der Hoffnung, dass Organoide als wichtiger Bestandteil der personalisierten Medizin nicht nur technisch und rechtlich, sondern auch tatsächlich eingesetzt werden können.

\subsection{Literaturverzeichnis}

Amato, F. et al. (2020): Cholangiocarcinoma disease modelling through patients derived organoids. In: Cells 9(4): 832.

Arning, F. (2018): Gesundheitsdatenschutz. In: Moos, F. et al. (Hrsg.): Die neue Datenschutz-Grundverordnung. De Gruyter, Berlin/Boston: 688-714, Rn. $179 \mathrm{ff}$.

Artegiani, B. et al. (2020): Fast and efficient generation of knock-in human organoids using homology-independent CRISPR-Cas9 precision genome editing. In: Nat Cell Biol. 22(3): 321-331.

Article 29 Working Party (2016): Guidelines on consent under regulation 2016/679. Unter: https://ec. europa.eu/newsroom/article29/item-detail.cfm?item_id=623051 [24.06.2020].

Bartfeld, S./Clevers, H. (2018): Aus Stammzellen abgeleitete Organoide und ihre Bedeutung für die biomedizinische Forschung und Therapie. In: Zenke, M. et al (Hrsg.): Stammzellforschung. Aktuelle wissenschaftliche und gesellschaftliche Entwicklungen. Nomos, Baden-Baden: 90-95.

BfDI (2020): BfDI zu Folgen der Gesetzgebung des PDSG. Unter: https://www.bfdi.bund.de/DE/Infoth ek/Pressemitteilungen/2020/20_BfDI-zu-PDSG.html [08.09.2020].

Bischoff, C. (2019): Datenschutz im Rahmen klinischer Prüfungen - das Zusammenspiel der Verordnung über klinische Prüfungen und der Datenschutz-Grundverordnung. In: PharmR 6: 265-272.

Boers, S. N. et al. (2016): Organoid biobanking: identifying the ethics. In: EMBO Rep. 17(7): 938-941.

Boers, S. N. et al. (2019): Organoids as hybrids: ethical implications for the exchange of human tissues. In: Med Ethics 45(2): 132-139.

Bundesgesundheitsministerium (2020): Entwurf eines Gesetzes zum Schutz elektronischer Patientendaten in der Telematikinfrastruktur. Unter: https://www.bundesgesundheitsministerium.de/filea dmin/Dateien/3_Downloads/Gesetze_und_Verordnungen/GuV/P/Gesetzentwurf_Patientendaten -Schutz-Gesetz_-_PDSG.pdf [14.06.2020]. 
Committee of Ministers (2019): Recommendation CM/Rec(2019)2 of the Committee of Ministers to member states on the protection of health-related data. Unter: https://rm.coe.int/CoERMPublicCo mmonSearchServices/DisplayDCTMContent?documentId=090000168093b26e [14.06.2020].

Datenschutzkonferenz (2019): Beschluss der 97. Konferenz der unabhängigen Datenschutzaufsichtsbehörden des Bundes und der Länder zu Auslegung des Begriffs „bestimmte Bereiche wissenschaftlicher Forschung“ im Erwägungsgrund 33 der DS-GVO. Unter: https://www.datenschutzko nferenz-online.de/media/dskb/20190405_auslegung_bestimmte_bereiche_wiss_forschung.pdf [16.06.2020].

Deutsche Referenzzentrum für Ethik in den Biowissenschaften (2020): Organoide. Unter: http://www .drze.de/im-blickpunkt/stammzellen/module/organoide/?searchterm=organoid [15.06.2020].

EDPB = European Data Protection Board (2019): Stellungnahme 3/2019 zu den Fragen und Antworten zum Zusammenspiel der Verordnung über klinische Prüfungen und der Datenschutz-Grundverordnung (DSGVO) (Art. 70 Abs. 1 b). Unter: https://edpb.europa.eu/sites/edpb/files/files/file1/ed pb_opinionctrq_a_final_de.pdf [16.06.2020].

Europäisches Patentamt (2020): Hans Clevers (The Netherlands). Finalist for the European Inventor Award 2017. Unter: https://www.epo.org/learning-events/european-inventor/finalists/2017/clev ers.html [15.06.2020].

Kaye, J. et al. (2014): Dynamic consent: a patient interface for twenty-first century research networks. In: European Journal of Human Genetics 23: 141-146.

Kim, M. et al. (2019): Patient-derived lung cancer organoids as in vitro cancer models for therapeutic screening. In: Nat Commun 10: 3991. https://doi.org/10.1038/s41467-019-11867-6 [15.06.2020].

Krawczak, M./Weichert, T. (2017): Vorschlag einer modernen Dateninfrastruktur für die medizinische Forschung in Deutschland (Version 1.9). Unter: https://www.uni-kiel.de/medinfo/document s/TWMK\%20Vorschlag\%20DInfMedForsch\%20v1.9\%20170927.pdf [08.09.2020].

Kühling, J./Buchner, B. (2018): Datenschutz-Grundverordnung/Bundesdatenschutzgesetz: DS-GVO / BDSG. 2. Aufl. C.H. Beck, München.

Lancaster, M. A./Knoblich, J. A. (2014): Organogenesis in a dish: Modeling development and disease using organoid technologies. In: Science 345(6194): 1247125.

Listl-Nörr, S. (2018): AMG § 40 Allgemeine Voraussetzungen der klinischen Prüfung. In: Spickhoff, A. (Hrsg.): Medizinrecht. C. H. Beck, München.

Maher, N. A. et al. (2019): Passive data collection and use in healthcare: A systematic review of ethical issues. In: International Journal of Medical Informatics 129: 242-247. Unter: https:// www.sciencedirect.com/science/article/pii/S1386505619302527?via\%3Dihub [15.06.2020].

Max-Planck-Gesellschaft (2020): Ein künstliches Lungenmodell als Testsystem für ein Corona-Medikament. Unter: https://www.mpg.de/14672877/alveolaere-epithelien-als-modell-fuer-eine-coron a-infektion [15.06.2020].

Medizininformatik-Initiative (2019): Stellungnahme der AG Consent der Medizininformatik-Initiative zu patientenindividueller Datennutzungstransparenz und Dynamic Consent. Unter: https://www. medizininformatik-initiative.de/sites/default/files/2019-09/MII_AG-Consent_Stellungnahme-Con sent-Modelle_v05.pdf [17.06.2020]. 
Medizininformatik-Initiative (2020): Handreichung zur Anwendung der national harmonisierten Patienteninformations- und Einwilligungsdokumente zur Sekundärnutzung von Patientendaten. AG Consent der Medizininformatik-Initiative (MII). Unter: https://www.datenschutzkonferenz-on line.de/media/pm/MII_AG-Consent_Handreichung_v0.9d.pdf [17.06.2020].

Moos, F. et al. (2018): Zulässigkeit der Verarbeitung personenbezogener Daten. In: Moos, F. et al. (Hrsg.): Die neue Datenschutz-Grundverordnung. De Gruyter, Berlin/Boston: 77-140, Rn. 107.

Mörl, K. (2019): Trendbericht Biochemie Teil 4: Organoide. In: Nachrichten aus der Chemie 67(7-8): 61-64.

OECD (2017): Recommendation on health data governance. Unter: http://www.oecd.org/health/heal th-systems/Presentation-Health-Data-Governance-Recommendation.pdf [24.06.2020].

Otlowski, M. (2009): Developing an appropriate consent model for biobanks: in defence of 'broad' consent. In: Kaye, S. (Hrsg.): Principles and Practise in Biobank Governance. Aschgate Publishing Company, Burlington: 79-92.

Pitzschke, A. (2017): Vom Organmodell zum Organersatz. Organoide. In: Laborjournal.de, Online Publikation 11.10.2017. Unter: https://www.laborjournal.de/rubric/methoden/methoden/v182.php [15.06.2020].

Rossen, N. S. et al. (2020): Injectable therapeutic organoids using sacrificial hydrogels. In: iScience 23(5): 101052.

Rowe, R. G./Daley, G. Q. (2019): Induced pluripotent stem cells in disease modelling and drug discovery. In: Nature Reviews Genetics 20: 377-388.

Sachs, N. et al. (2019): Long-term expanding human airway organoids for disease modelling. In: EMBO J. 38(4): e100300.

Schaar, K. (2017): Anpassung von Einwilligungserklärungen für wissenschaftliche Forschungsprojekte. Die informierte Einwilligung nach der DS-GVO und den Ethikrichtlinien. Unter: http://scientifi cdata.de/images/Artikel/ZD-05-2017---Beitrag-Schaar.pdf [24.06.2020].

Schanz, P./Wolff, H. A. (2017): Das neue Datenschutzrecht. C.H. Beck, München.

Ubink, I. (2019): Organoids from colorectal peritoneal metastases as a platform for improving hyperthermic intraperitoneal chemotherapy. In: BJS 106: 1404-1414, Online Publikation 14.06.2019. Unter: https://bjssjournals.onlinelibrary.wiley.com/doi/epdf/10.1002/bjs.11206 [15.06.2020].

van de Wetering, M et al. (2015): Prospective derivation of a living organoid biobank of colorectal cancer patients. In: Cell 161(4): 933-945.

WMA = The World Medical Association (2016): WMA Declaration of Taipei on ethical considerations regarding health databases and biobanks. Unter: http://www.ak-med-ethik-komm.de/docs/WMA -Declaration-of-Tapei-2016.pdf [24.06.2020].

Wolf, C. (2020): Mit Miniorganen zum passenden Medikament. In: Spektrum.de, Online Publikation 12.02.2020. Unter: https://www.spektrum.de/news/kuenstliche-mini-organe-sollen-die-wirkung -von-medikamenten-vorhersagen/1703520 [15.06.2020]. 